\title{
Simulated Blocking Potassium Channels Medication on Variant Mutant SCN5A Sodium Channels
}

\author{
JC Hsieh ${ }^{1}$, SK Lin ${ }^{1}$, WC Tzeng ${ }^{1}$, SM Shieh ${ }^{2}$ \\ ${ }^{1}$ Chung Hua University, Hsinchu, Taiwan \\ ${ }^{2}$ Wei-Gon Memorial Hospital, Miaoli, Taiwan
}

\begin{abstract}
Two gene-mutant sodium channels, 1795insD and I1768V, were considered to be the possible molecular markers in the initiation of long $Q T$ (LQT) syndrome. The $1795 i n s D$, which decreases the channel availability and enhances the inactivation, and $11768 \mathrm{~V}$, which increases the channel availability and enhances the rate of recovery from inactivation, have the ability to induce $L Q T$ regardless of their heterogeneous physical characteristics. However, their arrhythmic susceptibility with the use of certain antiarrhythmic medications has not yet been examined closely. In this study, the two mutant SCN5A channels were explored to elucidate the interactions among various potassium channels, IKI, IKr, and IKs with simulated antiarrhythmic medications by computer modeling. The two mutant SCN5A Markov models, adapted to fit into Rudy's ventricular cell model, performed numerical calculation by using cvode, an ODE solver, with $C$ code in a 4-node PC cluster. In this study, our previously developed S1-S2 protocol was used to investigate the cell excitability in simulated blocking potassium channel medication. The results are as follows: (1) by blocking IKI from $10 \%$ to $80 \%$, the needed injection charges to initiate an action potential for 1795insD were smaller than I1768V's; (2) by blocking $70 \% \mathrm{IKr}$ with current stimuli at the rate of $0.5 \mathrm{~Hz}$, the action potential of $11768 \mathrm{~V}$ at the $4^{\text {th }}$ beat began to display premature repolarization; and (3) by blocking $40 \%-50 \%$ IKs with current stimuli at the rates of $0.5 \mathrm{~Hz}$ and $1 \mathrm{~Hz}$ respectively, the action potential of $11768 \mathrm{~V}$ at the $3^{\text {rd }}$ beat and the $7^{\text {th }}$ beat began to display premature repolarization. Accordingly, the blockage of IKI could demonstrate both positive and negative effects on the two mutant SCN5A channels, as it may enhance or reduce the channel availability while increasing or decreasing the charge threshold. In addition, the blockage of IKs for I1768V might cause serious premature repolarization than the blockage of IKr.
\end{abstract}

\section{Introduction}

Recent studies have revealed that both mutant sodium channels, LQT 1795insD and I1768V, could result in clinical type III LQT syndromes $[1,2]$. What is known about the physical properties of the 1795insD is that (1) it could decrease the channel availability and that (2) it could enhance the inactivation. Meanwhile, what is known about the physical properties of $11768 \mathrm{~V}$ is that (1) it could increase the channel availability, and that (2) it could enhance the rate of recovery from inactivation [2]. The LQT in cellular level could display prolonged refractory. The class III antiarrhythmic drugs have been used as the major intervention on LQT. However, not much is known about the interactions between potassium channel blockers and mutant sodium channels. In this study, various potassium channel blockers, such as IK1, $\mathrm{IKr}$, IKs, were simulated by reducing the maximum conductance of channels. The changes of action potentials were observed under various rates of stimuli from $0.5 \mathrm{~Hz}$ to $1.5 \mathrm{~Hz}$ and various potassium blockers. The vulnerability of mutant sodium channels were measured by the initiation of wave oscillation, which could prolong the action potential duration.

\section{Methods}

\subsection{The model}

Two gene-mutant sodium channels, 1795insD and I1768V based on Markov modeling, were incorporated into Rudy phase II cell model. The cell model was used to simulate the membrane excitability and ionic current activity under whole cell voltage clamp mode and current clamp mode. The relationships between membrane voltage and ionic currents are shown in Equation (1):

$$
\frac{\partial V}{\partial t}=\frac{1}{C}\left(I_{i o n}+I_{s t}\right)
$$

Where $\mathrm{V}$ represents the membrane voltage, C represents the membrane capacitance, $\mathrm{I}_{\text {ion }}$ represents the summation of total ionic currents, and $I_{\text {sti }}$ represents the 
external current stimuli.

\subsection{I-V relationship}

To better understand the physical properties of the two mutant sodium channels, the current-voltage relationship simulation was performed on voltage clamp mode. In the same manner, the activation and inactivation curves of two mutant sodium channels were described by model simulation.

\subsection{Cell excitability}

To understand the interactions between potassium blockage and cell excitability under mutant sodium channels, the S1-S2 stimulus protocol was applied on the cell model to observe the possible changes of action potentials. Where S1 represents the conditional current stimulus to invoke action potential, $\mathrm{S} 2$ represents the test current stimulus.

\subsection{The vulnerability of arrhythmias}

To test the vulnerability of mutant sodium channel under various potassium blockers, the initiation of early after-depolarization was observed. In Figure 1, the initiation of early after-depolarization can prolong the action potential duration. Therefore, long QT interval can display on E.C.G [3].

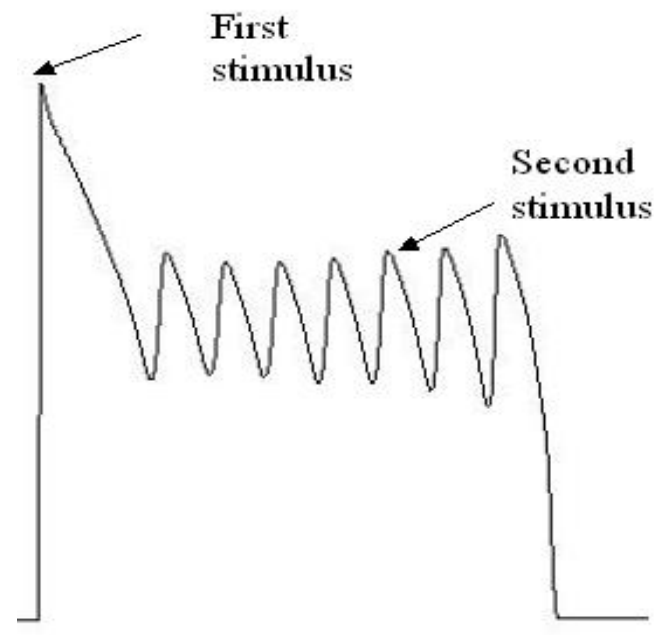

Figure 1. The initiation of early after-depolarization potential was used as the measurement of vulnerability.
The system differential equations of ionic channels described by Rudy's phase II model and equation (1) were sent to a partial differential equation solver, CVode, running on a 4-node Linux cluster at the lab of system physiology and biology in Chung Hua University.

\section{Results}

\subsection{Electrophysiological prosperities of sodium channel}

To understand the electrophysiological characteristics of three sodium channels including wild type, 1795insD and $11768 \mathrm{~V}$, the current -membrane voltage (I-V) relationship were measured by their activation and inactivation process. In Figure $2 \mathrm{~A}$, the three sodium channels had similar I-V relationship during channel activation.

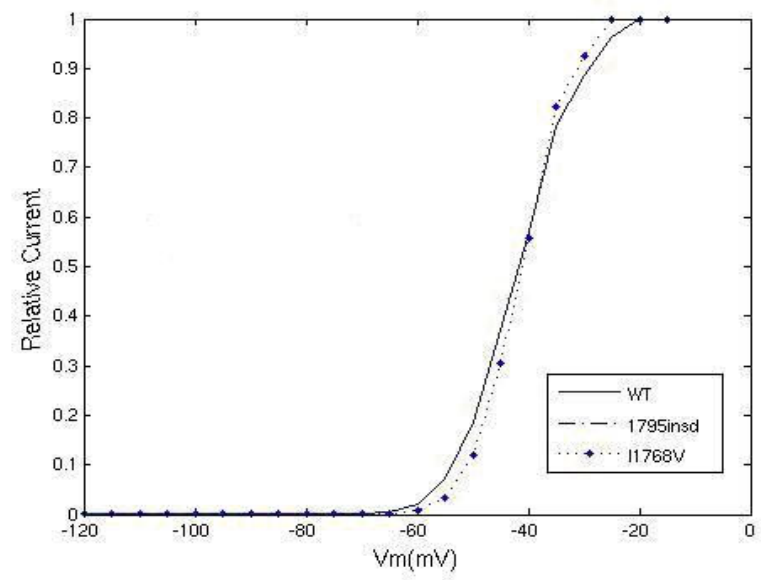

Figure 2A. Activation curves of three sodium channels including wild type, 1795insD and I1768V.

In the inactivation curve test, the three sodium channels showed large differences in electrophysiology. The inactivation cure of $1795 \mathrm{insD}$ was shifted to the left of wild type's; however, the inactivation curve of I1768V was shifted to the right. The result in Figure 2B indicated that the 1795 insD enhances the inactivation process.

\subsection{Numerical computation}




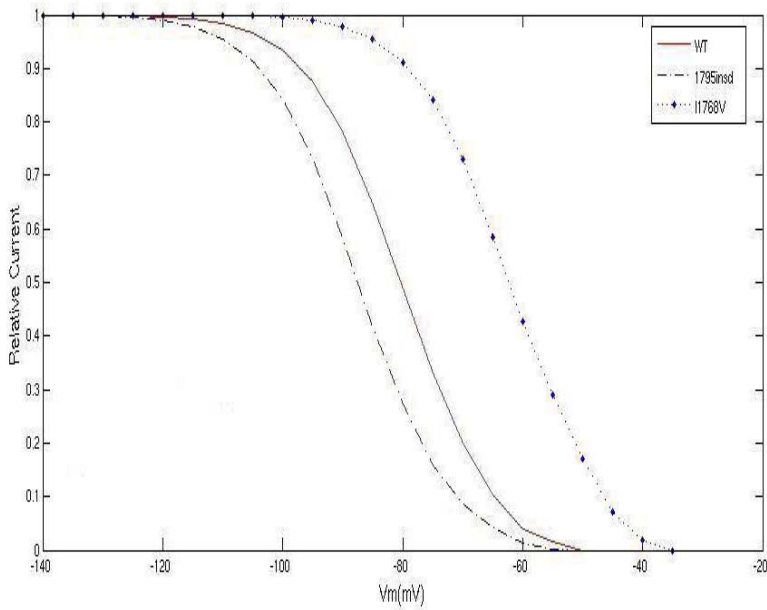

Figure 2B. The inactivation curves of three sodium channels: wild type, 1795insD, and I1768V.

As shown in Figure 3, the recovery rates from inactivation of three sodium channels were tested based on Rudy's protocol[], the I1768V on the left curve showed faster recovery rate than the wild type and 1795insD.

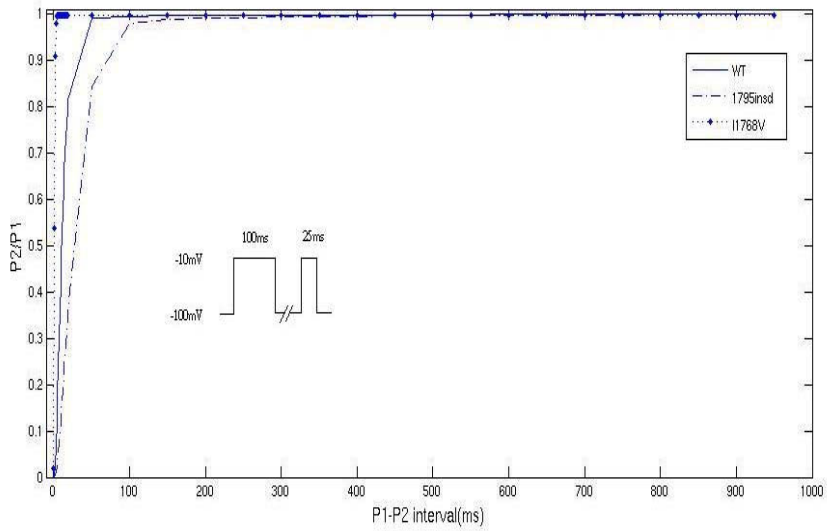

Figure 3. The curves of recovery rates from inactivation.

\subsection{Cell excitability under potassium blockers}

To better understand the interactions between cell excitability and potassium channel blockers, an S1-S2 current stimulus protocol was used to measure relative ratio of sodium currents by testing stimulus (S2) to the conditional stimulus (S1). In Figure 4A, the cell excitability was decreased with increased IK1 blockage in mutant and non-mutant sodium channels. However, I1798 showed higher excitability than 1795insD and the wild type sodium channel in IK1 blockage. As shown in Figure 4B, I1768V and 1795insD had the same degree of cell excitability despite of increased blockage of IKs from $40 \%$ to $70 \%$. In Figure 4C, the cell excitability of three sodium channels remained the same despite of increased $\mathrm{IKr}$ blockage. However, the .I1768V had highest excitability among the three sodium channels under the same blockage of IKr.

(A)

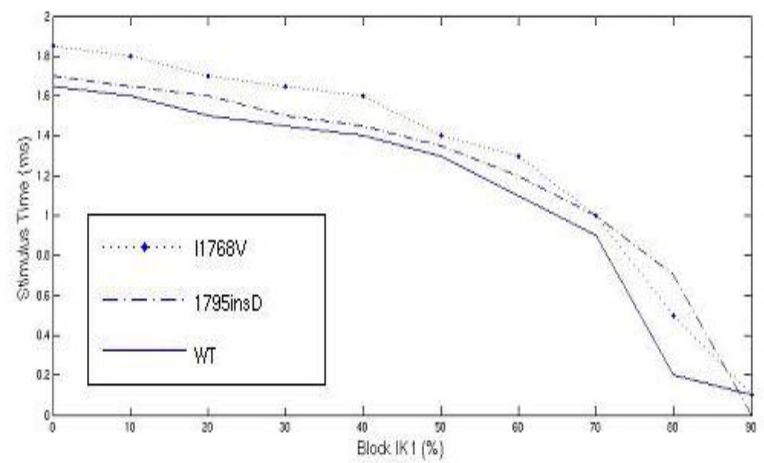

(B)

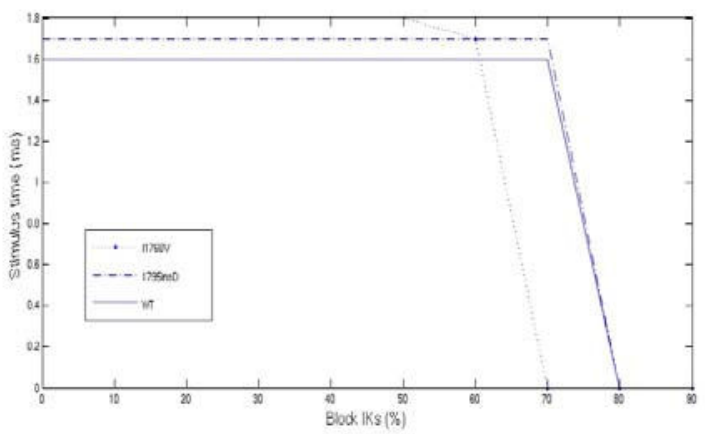

(C)

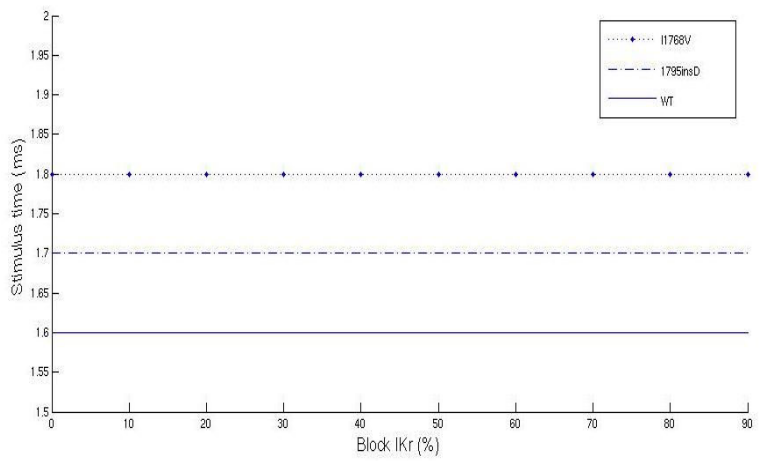

Figure 4 A,B,C. Cell excitability vs. various potassium channel blocker. The S1-S2 interval was 1500 ms. 


\subsection{Vulnerability vs Potassium Blockers}

The initiation of early afterdepolarization of action potentials was used as the vulnerability in this study. In Figure 5A, the stimulus interval was $1500 \mathrm{~ms}$ and Iks was $60 \%$ blockage, which resulted in early afterdepolarization and degenerated into arrhythmias on the incorporated I1768V cell model. In Figure 5B, the blockage of Iks was decreased to $50 \%$ and the stimulus interval was reduced to $1000 \mathrm{~ms}$, and the early afterdepolarization was initiated. In Figure 5C, non-sustained early afterdepolarization was observed on the $80 \%$ of blockage of Ikr at $1500 \mathrm{~ms}$ stimulus interval.

(A)

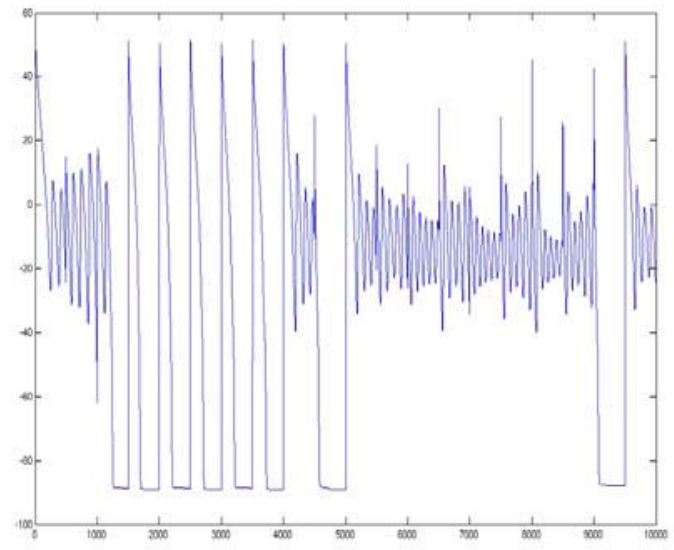

(B)

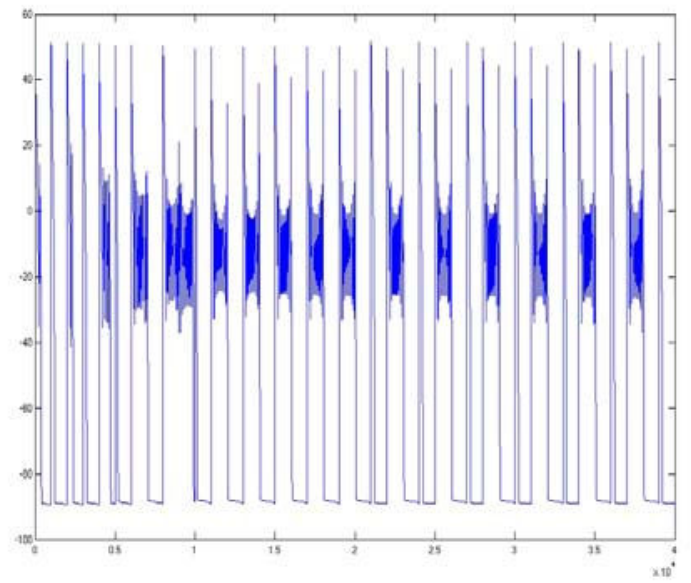

(C)

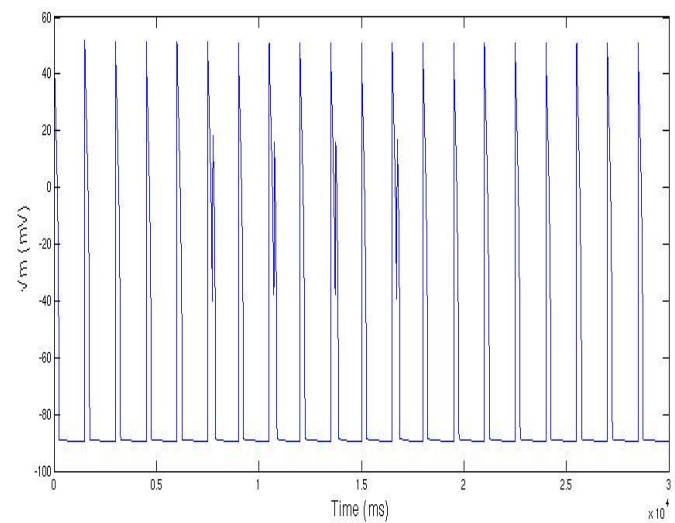

Figure 5 A,B,C. The initiation of early afterdepolarization of mutant $11768 \mathrm{~V}$ cell model under various IKs channel blockage.

\section{Discussion}

Based on out simulations, the needed injection charges to initiate an action potential for $1795 \mathrm{insD}$ were smaller than I1768V's by blocking IK1 from 10\% to $80 \%$. Accordingly, the blockage of IK1 could demonstrate both positive and negative effects on the two mutant SCN5A channels, as it may enhance or reduce the channel availability shown in Figure 4 while increasing or decreasing the charge threshold. Based on the simulations shown in Figure 5, it was found that the blockage of IKs for $11768 \mathrm{~V}$ might cause more serious premature afterdepolarization than the blockage of IKr.

\section{Acknowledgements}

This project was granted by NSC 94-2213-E-216-011 and NSC 94-2213-E-216-030 (National Science

Council, Taiwan) and supported by Chung Hua University, Hsin-chu, Taiwan.

\section{References}

[1] Rivolta I et, al. A novel $S C N 5 A$ mutation associated with long QT-3: altered inactivation kinetics and channel dysfunction. Physiol Genomics 2002;10: 191-197.

[2] Clancy CE, Rudy Y. $\mathrm{Na}^{+}$Channel Mutation that Causes Both Brugada and Long-QT Syndrome Phenotypes : A Simulation Study of Mechanism. Circulation 2002;105:1208-1213.

[3] Splawski I e,t al.: Variant of SCN5A Sodium Channel Implicated in Risk of Cardiac Arrhythmia. SCIENCE 2002; VOL 297:1333-1336. 